psychiatric disease. The risk of childhood autism following valproate use in women with epilepsy is lower compared with the overall risk. Exposures to carbamazepine, oxcarbazepine, lamotrigine and clonazepam monotherapy are not associated with increased risks of ASD and CA. The possible protective effects of folic acid ingestion during pregnancy are not evaluated. (Suren P, et al. JAMA 2013 Feb 13;309(6):570-7) (Schmidt RJ, et al. Am J Clin Nutr 2012 Jul;96(1):80-9).

The risks of valproate in women of childbearing potential (15-44 years) may outweigh the benefits. Given the evidence linking fetal valproate exposure to congenital malformations, cognitive impairments (Pediatr Neurol Briefs 2013 Apr;27(4):31), and autism, the use of valproate in potentially parous women should be minimized, restricted to cases with no available alternative treatment, and only introduced (in low dosage) after potential risks are fully explained. (Meador KJ, Loring DW. Editorial. Risks of in utero exposure to valproate. JAMA 2013 Apr 24;309(16):1730-1).

Fetal Growth and Risk of Autism. Investigators at the University of Manchester, UK, and other centers studied the relationship between fetal growth and autistic spectrum disorder (ASD). Deviance in fetal growth at either extreme and preterm birth are strong independent risk factors for later ASD, especially with intellectual disabilities. Genetic and environmental factors might influence fetal growth and predispose to ASD. (Abel KM, et al. Deviance in fetal growth and risk of autistic spectrum disorder. Am J Psychiatry 2013 Apr 1;170(4):391-8).

\title{
ANTIEPILEPTIC DRUG DISPOSITION IN PREGNANCY
}

Investigators at the Karolinska Institute, Stockholm, Sweden; and centers in Oslo, Norway, and Milan, Italy, reviewed the literature on gestational effects on pharmacokinetics of older and newer antiepileptic drugs (AEDs). Absorption, distribution, metabolism, and elimination may be affected depending on the type of AED. A pronounced decline in serum concentrations is seen for AEDs eliminated by glucuronidation (UGT), especially lamotrigine. Serum levels of AEDs cleared mainly through the kidneys, e.g. levetiracetam, decline significantly. In contrast, carbamazepine is affected only marginally by pregnancy. Data on newer drugs are either lacking or vary widely: pregabalin, lacosamide, retigabine, and eslicarbazepine acetate. In general, declining serum concentrations in pregnancy are associated with deterioration in seizure control. AED serum monitoring and dose adjustment based on falling levels may be important, particularly when a patient's levels are titrated to the lowest effective AED dose and serum concentration before pregnancy. (Tomson T, Landmark CJ, Battino D. Antiepileptic drug treatment in pregnancy: Changes in drug disposition and their clinical implications. Epilepsia 2013 Mar;54(3):405-14). (Response: Torbjorn Tomson, Department of Neurology, Karolinska University Hospital, Stockholm, Sweden. E-mail: trobjorn.tomson@karolinska.se).

COMMENT. Pharmacokinetic changes during pregnancy are rapid and labile and have a profound effect on the management of epilepsy. The risks of uncontrolled seizures are weighed against potential teratogenic effects of AEDs. 